

\title{
Avaliação de empresas aéreas com modelo de análise envoltória de dados clusterizado pela escala de operação
}

Ariane Lima de Moura+; Ana Paula dos Santos Rubem; João Carlos Correia Baptista Soares de Mello Universidade Federal Fluminense, Niterói, Brasil

\section{Article Info}

Palavras-chave: análise envoltória de dados clusterização

clusters dinâmicos

clusters estáticos

transporte aéreo

Submitted 26 Dec 2014; received in revised form 19 Jun 2015 accepted 12 Jul 2015.

Licensed under

Creative Commons

CC-BY 3.0 BR.

\section{Resumo}

Neste artigo, realiza-se uma análise comparativa dos resultados de eficiência obtidos usando duas abordagens preexistentes de Análise Envoltória de Dados em relação à metodologia aqui proposta, que representa uma alternativa para lidar com diferenças de escala. Para tanto, é conduzido um estudo sobre o desempenho operacional das empresas brasileiras de transporte aéreo de passageiros, quanto ao uso da frota. A proposta combina clusterização estática ao modelo para retornos constantes de escala, e compensação das diferenças de escala entre os clusters. A inovação está na adaptação de técnica, a princípio, idealizada para lidar com a não-homogeneidade, ao tratamento de diferenças de escala. Os resultados revelam que a metodologia proposta aumenta a discriminação entre as empresas, havendo unidades 100\% eficientes nas abordagens preexistentes testadas, cuja eficiência foi reduzida, ocasionando menos empates. Outro aspecto relevante é que a metodologia proposta apresenta vantagens comparativas em relação as alternativas existentes na literatura.

+ Corresponding author. Rua Passo da Pátria, 156, São Domingos, Niterói, RJ 24210-240, Brasil.

E-mail address: ariane.moura@yahoo.com.br.

\section{Introdução}

O objetivo deste artigo é adaptar uma técnica originalmente idealizada para lidar com a não-homogeneidade das DMUs (Gomes et al., 2012) ao tratamento de diferenças de escala de operação. Para tanto, será usado o modelo de Análise Envoltória de Dados (DEA, Data Envelopment Analysis), introduzido por Charnes, Cooper e Rhodes (CCR: Charnes et al., 1978), combinado à clusterização estática, seguidos de uma solução para compensação de escala entre clusters. 0 presente artigo expande trabalho preliminar apresentado por Rubem et al. (2014).

A motivação para essa adaptação deriva do fato de a extensão do modelo CCR, desenvolvida por Banker, Charnes e Cooper (BCC: Banker et al., 1984), e que se propõe a tratar fatores de escala (o CCR assume que todas as unidades produtivas operam em escala ótima), vir sendo alvo de críticas, detalhadas a seguir. 0 propósito do modelo BCC é permitir que uma unidade possa ser eficiente, operando sob retornos crescentes, constantes ou decrescentes de escala. Mas, esse modelo é muito usado para comparar unidades que operam sob escalas de produção diferentes, trazendo desvantagens: classificação inadequada de unidades como eficientes (Ali, 1993; Maciel et al., 2014); elevação nas pontuações de eficiência, reduzindo a discriminação (Rubem et al., 2014); obtenção de pontuações de eficiência distintas, dependendo da orientação adotada para o modelo (Benício e Soares de Mello, 2014); e eficiências negativas implícitas (Soares de Mello et al., 2013).

Dentro desse contexto, a contribuição deste trabalho é propor uma alternativa, que além de não incorrer nas desvantagens acima mencionadas, permita a comparação global das unidades produtivas, o que não é possível nas abordagens que combinam clusterização estática ao modelo CCR ou mesmo, eventualmente, na clusterização dinâmica (Golany e Thore, 1997).

Para avaliação da abordagem proposta, será conduzido um estudo de caso sobre o desempenho operacional das empresas brasileiras de transporte aéreo de passageiros, no que se refere ao uso da frota. Dentre as quais, há algumas regionais, que operam pequena frota de aviões com baixa capacidade (e.g., Abaeté, Meta). Outras que operam apenas voos domésticos, usando frota com um número maior de aeronaves com capacidade de transporte intermediário (e.g., Avianca, Azul, Trip). Há, ainda, empresas que operam rotas nacionais e internacionais, usando frota formada por modelos com grande capacidade (e.g., Gol/Varig, TAM). Logo, não parece razoável supor condições homogêneas de escala.

Os resultados obtidos validam a abordagem proposta, indicando maior discriminação das unidades produtivas (empresas aéreas) avaliadas, tanto em relação ao modelo BCC, como em relação à clusterização dinâmica integrada ao modelo CCR.

0 artigo está assim organizado: a Seção 1 contém uma breve revisão de DEA, clusterização e tratamento de fatores de escala. Na Seção 2, descreve-se a metodologia proposta. A Seção 3 apresenta a modelagem do problema, os resultados das abordagens usadas e uma análise comparativa. A última seção traz as conclusões e sugestão de trabalho futuro. 


\section{Referencial teórico}

\subsection{DEA}

DEA é uma abordagem não-paramétrica, baseada em programação matemática, que permite análises comparativas de unidades produtivas, chamadas de DMUs (Decision-Making Units). Essas DMUs convertem, em quantidades variadas, um conjunto de insumos em múltiplos produtos ou serviços. As DMUs podem ser qualquer tipo de organização, desde que atuem sob as mesmas condições e tenham autonomia na tomada de decisões. Os insumos e produtos devem ser os mesmos para todas as unidades. Em suma, a premissa básica em DEA é a homogeneidade das DMUs em análise (Angulo-Meza, 1998).

Outro aspecto relevante é a proporcionalidade entre os insumos consumidos e os produtos produzidos no processo avaliado. Os modelos mais usados são os já citados CCR, que lida com esta relação de forma estritamente proporcional; e BCC, que se propõe a incorporar fatores de escala, ou seja, um incremento no insumo não conduz a um incremento proporcional na produção. Quando as DMUs avaliadas operam em escalas muito diferentes, comumente, recorre-se ao modelo BCC.

Uma alternativa consiste em combinar o modelo CCR a técnicas de clusterização. Embora, a clusterização em DEA, geralmente, tenha o propósito de segregar DMUs não-homogêneas (Gomes et al., 2012), aqui, ela é usada para segregar DMUs operando sob escala de produção diferentes, a fim de permitir o uso do modelo CCR, no lugar do BCC, sem negligenciar as diferenças de escala. Sendo assim, agrupa-se as DMUs com escalas semelhantes e faz-se a análise de cada cluster separadamente. A desvantagem desse tipo de abordagem é que, como DEA se baseia em uma medida relativa (eficiência), uma comparação global entre DMUs de clusters distintos não é válida, a menos que se aplique uma solução de homogeneização (veja, e.g., em Bertoloto e Soares de Mello, 2011; Gomes et al., 2012), estratégia também adotada neste artigo.

Outra forma de tentar vencer essa limitação, é recorrer à já mencionada abordagem de Golany e Thore (1997), detalhada na subseção seguinte, que integra a clusterização dinâmica ao modelo CCR, e que, em geral, permite a comparação global das DMUs, ainda que de forma indireta, desde que satisfeitas certas condições (Andrade et al., 2014).

\subsection{Análise de clusters}

A análise de cluster congrega diferentes técnicas para avaliar a semelhança entre as unidades de um conjunto, segundo critérios predefinidos, a fim de formar grupos de similaridade. Sendo assim, os grupos gerados (ou clusters) são nãohomogêneos entre si, mas internamente homogêneos. Um dos algoritmos de clusterização estática mais usados é o K-means (MacQueen, 1967), dada sua facilidade de implementação. Seu objetivo é particionar um conjunto em k subconjuntos disjuntos, tal que a soma dos quadrados das distâncias das unidades (aqui DMUs) até o centro dos clusters seja minimizada. 0 valor k (número de clusters desejados) é definido a priori e serve de entrada para o algoritmo.

Já a clusterização dinâmica (Golany e Thore, 1997), cria grupos em que cada unidade (DMU) é o ponto central de um cluster específico, ajustando uma vizinhança em torno de cada unidade. Nesse caso, os clusters gerados não são, necessariamente, disjuntos (vide Figura 1(a)). Por essa razão, é possível realizar uma análise mais abrangente (veja, e.g., Andrade et al., 2014) do que a permitida em um esquema de clusterização estática convencional.

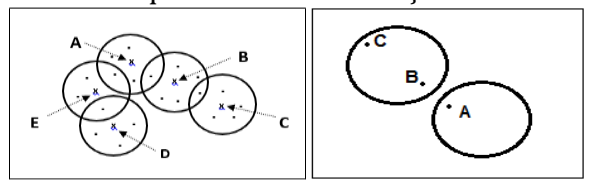

(a)

(b)

Figura 1 - Clusterização (a) dinâmica e (b) estática

A abordagem dinâmica visa superar o fato de a clusterização estática possibilitar que uma unidade (B) situada na extremidade de um cluster seja mais próxima a uma unidade (A) situada em outro cluster do que de outra unidade (C) de seu próprio cluster (Andrade et al., 2014), conforme mostrado na Figura 1(b). Ademais, a abordagem estática pode gerar clusters muito pequenos (poucas unidades) para análises DEA tradicionais.

\section{Metodologia proposta: K-means, CCR e homogeneização de escala}

Em geral, as abordagens que combinam DEA e clusterização estática começam agrupando as DMUs, segundo critérios estruturais ou ambientais para, então, aplicar o modelo CCR separadamente em cada cluster. Essas abordagens costumam ser usadas para tratar a não-homogeneidade entre as DMUs e, neste caso, podem ser associadas tanto ao CCR como ao BCC. Neste artigo, o objetivo é propor uma metodologia inovadora, que combine o modelo CCR e clusterização estática para situações em que a não-homogeneidade se restrinja a diferenças nas escalas das DMUs, assegurando a comparação global entre clusters distintos. Para tanto, é necessária a aplicação de técnica de compensação para corrigir as diferenças de escala entre os clusters.

Dentre as alternativas disponíveis na literatura (e.g., Bertoloto e Soares de Mello, 2011), optou-se por uma solução expost (Gomes et al., 2012). O algoritmo aqui proposto obedece aos seguintes passos: (1) separar as DMUs em clusters homogêneos segundo a escala de operação, usando o método K-means; (2) rodar um modelo CCR específico para cada cluster gerado e selecionar as DMUs 100\% eficientes; (3) rodar um modelo CCR apenas com as DMUs eficientes de cada cluster, selecionadas no passo 2; (4) calcular as eficiências médias das DMUs do passo 3, separadas em seus clusters originais; (5) rodar um modelo CCR com todas as DMUs do conjunto de análise; e (6) usar as médias do passo 4 como fator de correção das eficiências de cada cluster, dividindo-se as eficiências do passo 5 pelas respectivas eficiências médias do passo 4. Em caso de eficiências maiores que um, fazer a normalização necessária. Diferentemente deste artigo, em Gomes et al. (2012) e Bertoloto e Soares de Mello (2011), a clusterização é feita usando apenas critérios estruturais das DMUs (i.e., sem auxílio de método matemático). 


\section{Aplicação}

\subsection{Modelagem DEA}

Por simplicidade, e assumindo que uma empresa aérea possa focar nos load factors (indicador que denota a capacidade efetivamente usada de uma aeronave) como base para o cálculo de seu desempenho operacional, neste estudo, tal como em trabalhos anteriores mencionados a seguir, a análise se restringe ao uso adequado da frota.

A otimização da frota evita a ociosidade das aeronaves, reduzindo custos operacionais. Mas, a frota de cada empresa pode englobar vários modelos de aeronave de diferentes fabricantes com capacidades de transporte distintas. Logo, em vez de usar o número de aeronaves operado por cada empresa como input, optou-se pelo peso máximo de decolagem (MCTOM, Maximum Certified Take-Off Mass) para o cômputo do input. Para tanto, somou-se os valores de MCTOM de cada aeronave da frota de uma dada empresa. Tal soma denota a capacidade de transporte da frota da empresa, em termos de carga e passageiros, sendo, portanto, usada como input no modelo CCR. Os trabalhos de Correia et al. (2011), Gomes Júnior et al. (2015), Pereira et al. (2012) e Silveira et al. (2012) usam o mesmo input para análise de eficiência de companhias aéreas.

No ramo de transporte aéreo de passageiros, o principal output das empresas é dado pela quantidade total de passageiros transportados multiplicada pelo número total de quilômetros voados por todas as aeronaves da frota (pax.km). Por tal valor ignorar outputs não associados a passageiros, o output tonelagem total de carga transportada multiplicada pelo total de quilômetros voados por toda frota (ton.km), também foi inserido na análise. Os trabalhos de Correia et al. (2011) e Gomes Júnior et al. (2015) usam os mesmos outputs.

Nesta modelagem, a ênfase em load factors negligencia inputs além da capacidade da aeronave (e.g., mão-de-obra, combustível). Ademais, a competitividade do mercado aumenta a relevância da qualidade e tempestividade do serviço. Todavia, esses fatores estão fora do escopo deste artigo, que se limita à avaliação operacional das empresas aéreas quanto à eficiência no uso da frota. Tal procedimento está de acordo com o entendimento de Cook et al. (2014), os quais destacam que quando do uso de DEA deve-se especificar claramente o propósito da avaliação. Para trabalhos dentro desse mesmo escopo, veja, e.g., Correia et al. (2011) e Gomes Júnior et al. (2015). Para modelagens alternativas, sob uma perspectiva mais abrangente, veja, e.g., Pereira et al. (2012) e Silveira et al. (2012).

Aqui, a orientação a input foi usada, já que o propósito é identificar as empresas que podem reduzir a capacidade de transporte de sua frota, sem prejuízo ao total de passageiros e carga transportados. Os dados referem-se aos anos de 2007 a 2010, e foram obtidos na página da Agência Nacional de Aviação Civil (ANAC). A análise começa em 2007, por ser o ano subsequente à grave crise no setor aéreo nacional, causada, principalmente, pela falência da Varig e pela queda de um avião da Gol. Logo, para uma maior consistência, optou-se por analisar anos com características semelhantes.

Adotou-se, ainda, uma abordagem longitudinal, que considera a mesma empresa como uma DMU separada em anos diferentes, assumindo que a tecnologia se manteve estável no período (Podinovski and Thanassoulis, 2007). A Tabela 1 apresenta os input e outputs de cada uma das 73 DMUs avaliadas, além das eficiências atinentes a cada passo da metodologia proposta na seção anterior, bem como as obtidas a partir do uso tradicional do modelo BCC e da clusterização dinâmica.

\subsection{Resultados}

No passo 1, aplicou-se o K-means $(\mathrm{k}=4)$ ao input "capacidade da frota", para gerar os clusters homogêneos quanto à escala. Porém, o uso tradicional do K-means, não asseguraria o atingimento do número mínimo de DMUs sugerido por Banker et al. (1989) para análises DEA (neste caso, 9 DMUs). Assim, a estratégia adotada consistiu em rodar o K-means e agregar os clusters gerados menores que 9, respeitada a ordenação das DMUs. As DMUs agregadas para formar tal cluster foram retiradas, e o K-means rodado novamente. 0 processo foi repetido até que todas as DMUs fossem alocadas a um cluster. 0 processo resultou nos 5 clusters exibidos na Tabela 1, que traz ainda as eficiências intra-cluster de cada DMU fruto da aplicação do modelo CCR separadamente para cada cluster (passo 2). Procedeu-se, então, ao passo 3, com a aplicação do mesmo modelo CCR, mas agora às 7 DMUs 100\% eficientes. A partir das eficiências individuais obtidas, calculou-se, as eficiências médias das DMUs do cluster de eficientes, considerando seus clusters originais (passo 4). 0 fato de apenas a DMU Azul_2009 ter sido 100\% eficiente no cluster de eficientes indica que apenas o cluster 4 opera em escala ótima, enquanto os demais clusters apresentam desvantagens de escala que influenciam negativamente no resultado, a despeito de outras ineficiências gerenciais.

No passo 5, aplica-se o mesmo modelo CCR a todas as DMUs do conjunto de análise. A seguir, no passo 6, as eficiências médias de cada cluster são usadas para corrigir as eficiências do passo anterior, dividindo-se as eficiências nele obtidas, pelas médias de cada cluster, considerando-se o cluster a que as DMUs foram originalmente designadas. Os resultados foram normalizados, pois havia eficiências maiores que um. Após a homogeneização de escala e subsequente normalização, a única DMU que se manteve 100\% eficiente foi a empresa Puma, em 2009, originalmente alocada ao cluster 2.

Em comparação ao BCC, a abordagem proposta discrimina melhor as DMUs, evitando as falsamente eficientes (Ali, 1993), gerando uma única DMU 100\% eficiente, contra 10 no BCC. A DMU Puma_2010 foi eficiente nas duas abordagens. No BCC, 2 DMUs eficientes foram assim classificadas por terem usado o menor nível de recursos (Sol_2009) ou produzido a maior quantidade em, ao menos, um dos outputs (TAM_2010). Vale lembrar que essa classificação inadequada resulta de propriedades matemáticas do modelo BCC, sendo uma das desvantagens que motivaram a metodologia alternativa aqui proposta, na qual as mesmas DMUs obtiveram resultados bem inferiores: 50\% para TAM_2010; e 22\% para Sol_2009. Além disso, na maioria dos casos, a metodologia proposta resultou em eficiências inferiores ao BCC.

Na clusterização dinâmica, os limites dos clusters também foram definidos a partir do input "capacidade da frota". A distância Euclidiana unidimensional foi usada como critério para a entrada de outras DMUs em cada um dos 73 clusters gerados, centrados em cada uma das DMUs do conjunto. Um aspecto relevante é que nesta aplicação a condição de inexistência de qualquer cluster disjunto em relação a todos os outros é satisfeita. Desse modo, até mesmo as eficiências resultantes de clusters não coincidentes são comparáveis entre si, ainda que indiretamente, podendo, inclusive, serem ordenadas (Andrade et al., 2014). Por fim, ressalta-se que a abordagem dinâmica resultou em 11 DMUs $100 \%$ eficientes: Azul, Noar, Puma e Rico, em 2010; Azul e Passaredo, em 2009; Passaredo e Webjet, em 2008; e Gol e Webjet, em 2007. 
Tabela 1 - Input e outputs praticados pelas DMUs e resultados de eficiencia frutos da aplicação de três abordagens distintas.

\begin{tabular}{|c|c|c|c|c|c|c|c|c|c|c|c|}
\hline \multirow{3}{*}{ DMU } & \multirow{3}{*}{$\begin{array}{l}\text { Capacidade } \\
\text { da Frota (t) }\end{array}$} & \multirow{3}{*}{$\begin{array}{c}\text { Pax.km } \\
\text { (milhares) }\end{array}$} & \multirow{3}{*}{$\begin{array}{c}\text { Ton.km } \\
\text { (milhares) }\end{array}$} & \multirow{3}{*}{ Cluster } & & & & Efici & ncia & & \\
\hline & & & & & & Abo & dagem 1 & roposta & & & \\
\hline & & & & & $\begin{array}{c}\text { Passos } \\
1-2 \\
\end{array}$ & $\begin{array}{c}\text { Passo } \\
3 \\
\end{array}$ & $\begin{array}{c}\text { Passo } \\
4 \\
\end{array}$ & $\begin{array}{c}\text { Passo } \\
5 \\
\end{array}$ & $\begin{array}{l}\text { Passo 6 } \\
\text { (norm.) }\end{array}$ & BCC & $\begin{array}{l}\text { Cluster } \\
\text { Dinâmico }\end{array}$ \\
\hline Sol_09 & 3 & 1212 & 99 & 1 & $73 \%$ & - & $21 \%$ & $7 \%$ & $22 \%$ & $100 \%$ & $73 \%$ \\
\hline Mega_07 & 4 & 1072 & 313 & 1 & $54 \%$ & - & $21 \%$ & $11 \%$ & $36 \%$ & $100 \%$ & $54 \%$ \\
\hline Sol_10 & 7 & 2245 & 186 & 1 & $67 \%$ & - & $21 \%$ & $6 \%$ & $20 \%$ & $53 \%$ & $67 \%$ \\
\hline Noar_10 & 10 & 5004 & 1426 & 1 & $100 \%$ & $21 \%$ & $21 \%$ & $21 \%$ & $66 \%$ & $51 \%$ & $100 \%$ \\
\hline Abaete_10 & 11 & 1933 & 161 & 1 & $34 \%$ & - & $21 \%$ & $3 \%$ & $10 \%$ & $31 \%$ & $34 \%$ \\
\hline Abaete_09 & 12 & 1645 & 139 & 1 & $28 \%$ & - & $21 \%$ & $3 \%$ & $9 \%$ & $29 \%$ & $28 \%$ \\
\hline Abaete_08 & 12 & 1378 & 125 & 1 & $23 \%$ & - & $21 \%$ & $2 \%$ & $7 \%$ & $28 \%$ & $23 \%$ \\
\hline Team_0̄9 & 14 & 3476 & 268 & 1 & $51 \%$ & - & $21 \%$ & $5 \%$ & $15 \%$ & $27 \%$ & $51 \%$ \\
\hline Abaete_07 & 15 & 2492 & 227 & 1 & $33 \%$ & - & $21 \%$ & $3 \%$ & $10 \%$ & $24 \%$ & $36 \%$ \\
\hline Team_10 & 17 & 3084 & 238 & 1 & $37 \%$ & - & $21 \%$ & $3 \%$ & $11 \%$ & $22 \%$ & $79 \%$ \\
\hline Team_08 & 18 & 3480 & 287 & 1 & $39 \%$ & - & $21 \%$ & $4 \%$ & $12 \%$ & $21 \%$ & $44 \%$ \\
\hline Sete_0̄8 & 20 & 9075 & 1000 & 2 & $28 \%$ & - & $66 \%$ & $10 \%$ & $10 \%$ & $25 \%$ & $100 \%$ \\
\hline Sete_07 & 20 & 8057 & 873 & 2 & $25 \%$ & - & $66 \%$ & $9 \%$ & $9 \%$ & $24 \%$ & $88 \%$ \\
\hline Team_07 & 21 & 3087 & 262 & 2 & $9 \%$ & - & $66 \%$ & $3 \%$ & $3 \%$ & $17 \%$ & $32 \%$ \\
\hline Sete_09 & 23 & 10514 & 1044 & 2 & $28 \%$ & - & $66 \%$ & $9 \%$ & $9 \%$ & $22 \%$ & $13 \%$ \\
\hline Meta_10 & 27 & 20088 & 1787 & 2 & $45 \%$ & - & $66 \%$ & $14 \%$ & $14 \%$ & $25 \%$ & $89 \%$ \\
\hline Pumā_09 & 28 & 43 & 4 & 2 & $0,1 \%$ & - & $66 \%$ & $0,03 \%$ & $0,03 \%$ & $12 \%$ & $0,2 \%$ \\
\hline Puma_08 & 28 & 4172 & 434 & 2 & $9 \%$ & - & $66 \%$ & $3 \%$ & $3 \%$ & $14 \%$ & $24 \%$ \\
\hline NHT_07 & 28 & 12638 & 1127 & 2 & $28 \%$ & - & $66 \%$ & $9 \%$ & $9 \%$ & $20 \%$ & $62 \%$ \\
\hline Puma_07 & 28 & 9763 & 1013 & 2 & $22 \%$ & - & $66 \%$ & $7 \%$ & $7 \%$ & $18 \%$ & $56 \%$ \\
\hline Sete_10 & 29 & 16794 & 1619 & 2 & $36 \%$ & - & $66 \%$ & $12 \%$ & $12 \%$ & $22 \%$ & $70 \%$ \\
\hline Meta_09 & 30 & 20277 & 1820 & 2 & $42 \%$ & - & $66 \%$ & $13 \%$ & $13 \%$ & $23 \%$ & $83 \%$ \\
\hline Meta_08 & 30 & 37800 & 3984 & 2 & $9 \%$ & - & $66 \%$ & $27 \%$ & $27 \%$ & $36 \%$ & $85 \%$ \\
\hline Meta_07 & 30 & 24602 & 2706 & 2 & $51 \%$ & - & $66 \%$ & $18 \%$ & $18 \%$ & $28 \%$ & $68 \%$ \\
\hline Passaredo_07 & 30 & 47629 & 4726 & 2 & $98 \%$ & - & $66 \%$ & $32 \%$ & $32 \%$ & $42 \%$ & $100 \%$ \\
\hline AirMinas_08 & 32 & 18744 & 1679 & 2 & $37 \%$ & - & $66 \%$ & $11 \%$ & $11 \%$ & $21 \%$ & $72 \%$ \\
\hline AirMinas_07 & 32 & 12289 & 1160 & 2 & $24 \%$ & - & $66 \%$ & $8 \%$ & $8 \%$ & $17 \%$ & $56 \%$ \\
\hline NHT_O8 & 34 & 16678 & 1499 & 2 & $30 \%$ & - & $66 \%$ & $9 \%$ & $9 \%$ & $18 \%$ & $60 \%$ \\
\hline NHT_10 & 40 & 10425 & 874 & 2 & $16 \%$ & - & $66 \%$ & $5 \%$ & $5 \%$ & $13 \%$ & $38 \%$ \\
\hline NHT_09 & 40 & 15435 & 1278 & 2 & $24 \%$ & - & $66 \%$ & $7 \%$ & $7 \%$ & $15 \%$ & $48 \%$ \\
\hline AirMinas_09 & 43 & 20820 & 1726 & 2 & $30 \%$ & - & $66 \%$ & $9 \%$ & $9 \%$ & $16 \%$ & $58 \%$ \\
\hline Passaredo_08 & 54 & 86746 & 8532 & 2 & $100 \%$ & $33 \%$ & $66 \%$ & $33 \%$ & $33 \%$ & $38 \%$ & $100 \%$ \\
\hline Puma_10 & 59 & 86803 & 45216 & 2 & $100 \%$ & $100 \%$ & $66 \%$ & $100 \%$ & $100 \%$ & $100 \%$ & $100 \%$ \\
\hline Pantanal_09 & 92 & 77465 & 6544 & 3 & $36 \%$ & - & $42 \%$ & $16 \%$ & $25 \%$ & $19 \%$ & $36 \%$ \\
\hline Passaredo_09 & 95 & 221821 & 19283 & 3 & $100 \%$ & $44 \%$ & $42 \%$ & $44 \%$ & $69 \%$ & $47 \%$ & $100 \%$ \\
\hline Pantanal_08 & 101 & 79126 & 7296 & 3 & $34 \%$ & - & $42 \%$ & $15 \%$ & $24 \%$ & $18 \%$ & $36 \%$ \\
\hline Pantanal_07 & 102 & 93420 & 8679 & 3 & $40 \%$ & - & $42 \%$ & $18 \%$ & $28 \%$ & $21 \%$ & $42 \%$ \\
\hline Rico_08 & 124 & 50013 & 5252 & 3 & $18 \%$ & - & $42 \%$ & $8 \%$ & $13 \%$ & $11 \%$ & $21 \%$ \\
\hline Webjet_07 & 155 & 345579 & 35885 & 3 & $100 \%$ & $46 \%$ & $42 \%$ & $46 \%$ & $73 \%$ & $48 \%$ & $100 \%$ \\
\hline Trip_07 & 157 & 208626 & 22677 & 3 & $62 \%$ & - & $42 \%$ & $28 \%$ & $44 \%$ & $30 \%$ & $62 \%$ \\
\hline TAF_09 & 159 & 4826 & 37645 & 3 & $84 \%$ & - & $42 \%$ & $31 \%$ & $48 \%$ & $31 \%$ & $31 \%$ \\
\hline Rico_07 & 171 & 161617 & 17833 & 3 & $44 \%$ & - & $42 \%$ & $20 \%$ & $32 \%$ & $22 \%$ & $45 \%$ \\
\hline Rico_09 & 173 & 4270 & 366 & 3 & $1 \%$ & - & $42 \%$ & $0,5 \%$ & $1 \%$ & $2 \%$ & $2 \%$ \\
\hline Rico_10 & 182 & 634 & 51767 & 3 & $100 \%$ & $37 \%$ & $42 \%$ & $37 \%$ & $57 \%$ & $40 \%$ & $37 \%$ \\
\hline Pantanal_10 & 205 & 242946 & 20823 & 3 & $51 \%$ & - & $42 \%$ & $22 \%$ & $35 \%$ & $24 \%$ & $51 \%$ \\
\hline Passaredo_10 & 215 & 428593 & 37401 & 3 & $86 \%$ & - & $42 \%$ & $38 \%$ & $59 \%$ & $39 \%$ & $75 \%$ \\
\hline Trip_08 & 281 & 517235 & 51882 & 4 & $41 \%$ & - & $100 \%$ & $38 \%$ & $25 \%$ & $38 \%$ & $83 \%$ \\
\hline Azul_09 & 391 & 2110707 & 176730 & 4 & $100 \%$ & $100 \%$ & $100 \%$ & $100 \%$ & $66 \%$ & $100 \%$ & $100 \%$ \\
\hline TAF_07 & 399 & 167685 & 51366 & 4 & $29 \%$ & - & $100 \%$ & $18 \%$ & $12 \%$ & $18 \%$ & $100 \%$ \\
\hline Webjet_08 & 431 & 1180795 & 123580 & 4 & $63 \%$ & - & $100 \%$ & $57 \%$ & $38 \%$ & $58 \%$ & $63 \%$ \\
\hline TAF_08 & 432 & 77986 & 44866 & 4 & $23 \%$ & - & $100 \%$ & $16 \%$ & $10 \%$ & $16 \%$ & $99 \%$ \\
\hline Total_09 & 498 & 62230 & 49086 & 4 & $22 \%$ & - & $100 \%$ & $20 \%$ & $13 \%$ & $23 \%$ & $100 \%$ \\
\hline Total_07 & 510 & 225952 & 49665 & 4 & $22 \%$ & - & $100 \%$ & $15 \%$ & $10 \%$ & $15 \%$ & $71 \%$ \\
\hline Trip_09 & 512 & 851302 & 76279 & 4 & $33 \%$ & - & $100 \%$ & $32 \%$ & $21 \%$ & $32 \%$ & $61 \%$ \\
\hline Total_08 & 518 & 66507 & 41849 & 4 & $18 \%$ & - & $100 \%$ & $14 \%$ & $9 \%$ & $11 \%$ & $79 \%$ \\
\hline Total_10 & 542 & 61992 & 52740 & 4 & $22 \%$ & - & $100 \%$ & $21 \%$ & $14 \%$ & $27 \%$ & $100 \%$ \\
\hline Avianca_10 & 789 & 1856243 & 171288 & 4 & $48 \%$ & - & $100 \%$ & $46 \%$ & $30 \%$ & $47 \%$ & $48 \%$ \\
\hline Trip_10 & 824 & 1547564 & 136879 & 4 & $37 \%$ & - & $100 \%$ & $36 \%$ & $24 \%$ & $36 \%$ & $37 \%$ \\
\hline Avianca_09 & 829 & 1438350 & 131355 & 4 & $35 \%$ & - & $100 \%$ & $34 \%$ & $22 \%$ & $34 \%$ & $35 \%$ \\
\hline Oceanair_07 & 918 & 1079481 & 120141 & 4 & $29 \%$ & - & $100 \%$ & $25 \%$ & $17 \%$ & $26 \%$ & $46 \%$ \\
\hline Webjet_09 & 955 & 2522478 & 226507 & 4 & $53 \%$ & - & $100 \%$ & $51 \%$ & $34 \%$ & $52 \%$ & $53 \%$ \\
\hline Azul_10 & 1041 & 4306850 & 357500 & 4 & $77 \%$ & - & $100 \%$ & $77 \%$ & $51 \%$ & $87 \%$ & $77 \%$ \\
\hline Oceanair_08 & 1116 & 1464627 & 159498 & 4 & $32 \%$ & - & $100 \%$ & $28 \%$ & $19 \%$ & $29 \%$ & $32 \%$ \\
\hline Webjet_10 & 1322 & 4130647 & 360628 & 4 & $60 \%$ & - & $100 \%$ & $59 \%$ & $39 \%$ & $72 \%$ & $60 \%$ \\
\hline Gol_07 & 4689 & 20306538 & 2119062 & 5 & $100 \%$ & $90 \%$ & $90 \%$ & $90 \%$ & $66 \%$ & $100 \%$ & $100 \%$ \\
\hline$V R G_{-} 07$ & 6597 & 3831335 & 478611 & 5 & $16 \%$ & - & $90 \%$ & $13 \%$ & $10 \%$ & $19 \%$ & $21 \%$ \\
\hline Gol/VRG_08 & 8658 & 26296872 & 2753740 & 5 & $70 \%$ & - & $90 \%$ & $63 \%$ & $47 \%$ & $78 \%$ & $70 \%$ \\
\hline Gol/VRG_09 & 8774 & 26339094 & 2391779 & 5 & $69 \%$ & - & $90 \%$ & $58 \%$ & $43 \%$ & $100 \%$ & $69 \%$ \\
\hline Gol/VRG_10 & 9191 & 31402872 & 2917360 & 5 & $79 \%$ & - & $90 \%$ & $67 \%$ & $49 \%$ & $100 \%$ & $79 \%$ \\
\hline$T A M \_07$ & 9553 & 33445234 & 3806729 & 5 & $88 \%$ & - & $90 \%$ & $77 \%$ & $56 \%$ & $100 \%$ & $88 \%$ \\
\hline TAM_08 & 12541 & 40702300 & 4671559 & 5 & $82 \%$ & - & $90 \%$ & $71 \%$ & $52 \%$ & $100 \%$ & $82 \%$ \\
\hline TAM_09 & 14028 & 44570946 & 4341646 & 5 & $73 \%$ & - & $90 \%$ & $64 \%$ & $47 \%$ & $91 \%$ & $73 \%$ \\
\hline TAM_10 & 15114 & 51712453 & 5010977 & 5 & $79 \%$ & - & $90 \%$ & $68 \%$ & $50 \%$ & $100 \%$ & $79 \%$ \\
\hline
\end{tabular}




\section{Conclusão}

O objetivo de propor uma metodologia inovadora e realizar uma análise comparativa com outras duas abordagens DEA que se propõem a tratar a não-homogeneidade (aqui, restrita a diferenças de escala) das DMUs foi atingido. A proposta combinou clusterização estática (K-means modificado) com o modelo CCR e subsequente solução de homogeneização. Em relação aos resultados obtidos, a abordagem proposta discriminou melhor as DMUs avaliadas. Entretanto, ela parece penalizar os resultados de eficiência, uma vez que as pontuações de todas as DMUs ineficientes são menores que na abordagem dinâmica e no BCC. A não ser pelos empates da abordagem dinâmica e BCC, as ordenações finais obtidas nas três abordagens são semelhantes. Pode-se concluir, então, que, se o objetivo for a ordenação das DMUs, os resultados deste estudo sugerem que a abordagem proposta apresenta a vantagem comparativa de gerar uma ordenação final com menos empates. Não obstante, as limitações típicas das abordagens que combinam DEA e clusterização estática foram identificadas: (a) necessidade de modificação do K-means para atingimento do tamanho mínimo no cluster; (b) DMUs situadas no limite dos clusters são mais próximos de uma DMU de outro cluster do que de outra de seu próprio cluster, gerando comparações injustas; (c) impossibilidade de comparação global entre DMUs, a menos que se recorra à solução de homogeneização. Uma possibilidade de trabalho futuro consiste em utilizar um método de alternativo de clusterização estática, buscando superar as dificuldades encontradas com o K-means, principalmente, no que se refere ao tamanho dos clusters gerados.

\section{Referências}

Ali, A.I. (1993). Streamlined computation for data envelopment analysis. European Journal of Operational Research, 64(1), 61-67.

Andrade, F.V.S., Andrade, G.N., \& Soares de Mello, J.C.C.B. (2014) Avaliação de investimento dos times de 3 ligas europeias de futebol com modelos DEA e clusters dinâmicos. Simpósio Brasileiro de Pesquisa Operacional, Salvador, Brasil.

Lins, M.P., Angulo-Meza, L. (2000) Análise envoltória de dados e perspectivas de integração no ambiente de apoio à decisão. Rio de Janeiro (Brasil): Editora da COPPE/UFRJ.

Banker, R.D., Charnes, A., \& Cooper, W.W. (1984). Some models for estimating technical scale inefficiencies in data envelopment analysis. Management Science, 30(9), 1078-1092.

Banker, R.D., Charnes, A., Cooper, W.W., Swarts, J., \& Thomas, D. (1989). An introduction to data envelopment analysis with some models and their uses. Research in Governmental and Non-Profit Accounting, 5, 125-163.

Bertoloto, R.F., \& Soares de Mello, J.C.C.B. (2011). Eficiência de portos e terminais privativos brasileiros com características distintas. Journal of Transport Literature, 5(2), 4-21.

Benício, J., \& Soares de Mello, J.C.C.B (2014). Retornos de escala em DEA: críticas ao BCC e novo modelo. Temiminós Revista Científica, 4(2), 42-61.

Charnes, A., Cooper, W.W., \& Rhodes, E. (1978). Measuring the efficiency of decision-making units. European Journal of Operational Research, 2(6), 429-444.

Cook, W.D., Tone, K., \& Zhu, J. (2014). Data envelopment analysis: prior to choosing a model, Omega, 44, 1-4.

Correia, T.C.V.D., Soares de Mello, J.C.C.B., \& Angulo-Meza, L. (2011). Assessment of Brazilian airlines technical efficiency: a study using data envelopment analysis and fuzzy sets, Produção, 21(4), 676-683.

Golany, B., \& Thore, S. (1997). Restricted best practice selection in DEA: An overview with a case study evaluating the socio-economic performance of nations. Annals of Operations Research 73, 117-140.

Gomes, E.G., Soares de Mello, J.C.C.B., \& Freitas, A.C.R. (2012). Efficiency measures for a non-homogeneous group of family farmers. Pesquisa Operacional, 32(3), 561-574.

Gomes Júnior, S.F., Rubem, A.P.S., Soares de Mello, J.C.C.B., \& Angulo-Meza, L. (2015). Evaluation of Brazilian airlines nonradial efficiencies and targets using an alternative DEA approach. International Transactions in Operational Research. DOI: 10.1111/itor.12162

Maciel, G. S., Lima, G.B.A., Angulo-Meza, L., \& Gomes Júnior, S.F. (2014). Avaliação de processos licitatórios de embarcações de apoio marítimo offshore com utilização de análise envoltória de dados. Journal of Transport Literature, 8(4), 329-349.

MacQueen, J.B. (1967). Some methods for classification and analysis of multivariate observations. Proceedings of 5th Berkeley Symposium on Mathematical Statistics and Probability: Volume I (pp. 281-304), Los Angeles (US): University of California Press.

Pereira, E.R., Silveira, J.Q., Chaves, M.C.C., \& Soares de Mello, J.C.C.B. (2012). Eficiência de empresas aéreas: uma análise baseada no modelo de Li \& Reeves. Journal of Transport Literature, 6(2), 105-123.

Podinovski, V.V., \& Thanassoulis, E. (2007). Improving discrimination in data envelopment analysis: some practical suggestions. Journal of Productivity Analysis, 28(1-2), 117-126

Rubem, A.P.S., Moura, A.L., Soares de Mello, J.C.C.B., \& Santos, B.M.F. (2014), Avaliação de empresas aéreas com modelo de análise envoltória de dados clusterizado pela escala de operação. Congresso Nacional de Pesquisa e Ensino em Transporte, Curitiba, Brasil.

Silveira, J.Q., Soares de Mello, J.C.C.B., \& Angulo-Meza, L. (2012). Identificação de benchmarks e anti-benchmarks para companhias aéreas usando modelos DEA e fronteira invertida. Produção, 22(4), 788-795.

Soares de Mello, J.C.C.B., Angulo-Meza, L., Silveira, J.Q., \& Gomes, E.G. (2013). About negative efficiencies in cross evaluation BCC input oriented models. European Journal of Operational Research, 229(3), 732-737.

\section{Abstract}

In this article, we carried out a comparative analysis of the efficiency results obtained using two existing approaches of DEA in relation to the methodology proposed here, which is an alternative to deal with differences of scale. For this, a study on the operational performance of the Brazilian air passenger transportation business is conducted. The proposal combines static clustering to the constant returns-to-scale model, and compensation scale differences among the clusters. The innovation relies on the adaption of a technique, originally designed to handle non-homogeneity to treat differences of scale. The results show that the proposed methodology increases the discrimination among companies, that is, some units that resulted $100 \%$ efficient in the existing approaches, had their efficiency reduced, resulting in less draws. Another relevant aspect is that the proposed methodology has comparative advantages over existing alternatives in the literature.

Key words: data envelopment analysis, clustering, dynamic clusters, static clusters, air transportation. 